\title{
Side effects of analgesia may significantly reduce quality of life in symptomatic multiple myeloma: a cross-sectional prevalence study
}

\author{
Sarah Sloot • Jason Boland • John A. Snowden • Yousef Ezaydi • Andrea Foster • \\ Alison Gethin • Tracy Green • Louise Chopra • Stans Verhagen • Kris Vissers • \\ Yvonne Engels • Sam H. Ahmedzai
}

Received: 16 April 2014 / Accepted: 21 July 2014 / Published online: 28 August 2014

(C) The Author(s) 2014. This article is published with open access at Springerlink.com

\begin{abstract}
Background Pain is a common symptom in patients with multiple myeloma (MM). Many patients are dependent on analgesics and in particular opioids, but there is limited information on the impact of these drugs and their side effects on health-related quality of life (HRQoL).
\end{abstract}

\section{S. Sloot}

Department of General Surgery, UMCG, GroningenHanzeplein, PO 30.001, 9713 GZ, Groningen, The Netherlands

\section{S. Sloot $(\bowtie)$}

Department of Cutaneous Oncology, Moffitt Cancer Center, 12902

Magnolia Dr, Tampa, FL 33612, USA

e-mail: sarahsloot@gmail.com

J. Boland

Hull York Medical School, University of Hull, Hull HU6 7RX, UK

J. A. Snowden · Y. Ezaydi · A. Foster

Department of Haematology, Sheffield Teaching Hospitals NHS

Foundation Trust, Sheffield S10 2JF, UK

A. Gethin $\cdot$ T. Green

North Trent Consumer Research Panel, c/o Academic Unit of Supportive Care, Department of Oncology, School of Medicine and Biomedical Science, The University of Sheffield, Beech Hill Road, Sheffield S10 2RX, UK

L. Chopra

Clinical Effectiveness Unit, Sheffield Teaching Hospitals NHS

Foundation Trust, 8 Beech Hill Road, Sheffield S10 2SB, UK

S. Verhagen $\cdot \mathrm{K}$. Vissers $\cdot$ Y. Engels

Department of Anesthesiology, pain and palliative medicine, UMC

St Radboud, PO 9101, 6500 HB Nijmegen, The Netherlands

S. H. Ahmedzai ( $\square)$

Academic Unit of Supportive Care, Department of Oncology, School of Medicine and Biomedical Science, The University of Sheffield,

Beech Hill Road, Sheffield S10 2RX, UK

e-mail: s.ahmedzai@sheffield.ac.uk
Method In a cross-sectional study, semi-structured interviews were performed in 21 patients attending the hospital with symptomatic MM on pain medications. HRQoL was measured using items 29 and 30 of the European Organisation for Research and Treatment of Cancer (EORTC) QLQ-C30.

Results Patients were able to recall a median of two (range 0-4) analgesics. They spontaneously identified a median of two (range 1-5) side effects attributable to their analgesic medications. Patients' assessment of HRQoL based on the EORTC QLQ-C30 questions 29/30 was mean 48.3 (95\% CI; 38.7-57.9) out of 100. Patients' assessment of their HRQoL in the hypothetical situation, in which they would not experience any side effects from analgesics, was significantly higher: 62.6 (53.5-71.7) $(t$ test, $p=0.001)$. Conclusion This study provides, for the first time, evidence that side effects of analgesics are common in symptomatic $\mathrm{MM}$ and may result in a statistically and clinically significant reduction of self-reported HRQoL.

Keywords Multiple myeloma · Quality of life · Opioid · Analgesics $\cdot$ Side effects

\section{Introduction}

Multiple myeloma (MM) has an incidence of approximately 60-70 cases per million inhabitants in the UK, with an average age at diagnosis of 70 years [1]. In recent years, the prognosis of symptomatic MM has improved dramatically owing to the advent of intensive treatments with haematopoietic stem cell transplantation and novel agents, including proteasome inhibitors and immunomodulatory drugs; as a result, increasing numbers of patients are surviving $>5-10$ years with the disease. Despite this, pain remains a 
common problem due to both osteolytic bone disease and increasingly treatment emergent peripheral neuropathy $[2,3]$.

The treatment of pain caused by multiple myeloma first starts with therapy directed at disease control, using cytotoxics, biological targeted drugs, radiotherapy and corticosteroids. Bone disease is also managed systemically using bisphosphonates, which are used principally to reduce future skeletal-related morbidity and may also reduce pain [4]. In addition, local pain control may be achieved using vertebroplasty or balloon kyphoplasty for vertebral fractures and surgical fixation for unstable long bones or spine $[5,6]$. Alongside these tumour-directed and supportive care measures, most patients with $\mathrm{MM}$ also receive pharmacological analgesic therapy, including opioid drugs $[2,7]$.

The recognition of specific side effects of prescribed analgesics is of great importance in the effective management of pain and optimisation of health-related quality of life (HRQoL) [8]. Weiss et al. (2001) showed that in a large US survey of 501 patients with terminal cancer, of whom $52 \%$ reported having 'moderate to severe pain', additional pain therapy was only sought by $29 \%$ of them. The reasons for not seeking additional therapy in terminal cancer patients who had been seen by their primary physician within the previous 4 weeks included: fear of addiction (37\%), fear of physical side effects (33\%), fear of mental side effects (34\%) and fear of pills and injections (29\%) [9]. Whilst some of these concerns represent real hazards of cancer pain treatment, it has been suggested that the adoption of a more multimodal pain strategy, with greater emphasis on the newer opioids, the use of non-opioids acting on the NMDA receptor and earlier use of nonpharmacological approaches, could reduce the emergence of adverse effects and thus improve patient experience [8].

Determining the right balance of analgesic efficacy against side effects is difficult as it depends on individual factors including patients' age, sex, general health, previous knowledge and health beliefs, as well as dosage [8, 10]. Only patients can weigh the positive effect of pain relief against the negative side effects. Therefore, the aim of this study was to determine whether patients with symptomatic MM could perceive a difference in their global health-related QoL, with and without analgesicrelated side effects.

\section{Methods}

Institutional approval

This study was approved by the Clinical Effectiveness Unit of the Sheffield Teaching Hospitals Foundation NHS Trust as a service evaluation.
Aim and objectives

Our main aim was to examine whether patients perceived an effect on their HRQoL, arising from the side effects they experienced from their pain medication.

Specific objectives were (1) to identify the medications used for pain control in symptomatic MM patients and to measure the extent to which patients could freely recall them, in contrast to recognising them from a prompt list; (2) to quantify the side effects of medications taken by MM patients and to identify to what extent they were able to attribute these side effects to analgesics; (3) to compare present HRQoL with hypothetical HRQoL if the identified side effects were eliminated.

\section{Population}

Patients diagnosed with symptomatic MM in a single tertiary centre were consecutively interviewed between October and December 2008. To be eligible for the interview, patients had to be 18 years or older and be able to communicate in English. In order to focus on patients with significant supportive care needs, patients who did not use any pain medication other than paracetamol and/or who were only on bisphosphonates or corticosteroids for bone disease were excluded. However, in the included patients, bisphosphonates were accounted for amongst the pain medication.

\section{Semi-structured interview}

A semi-structured interview was developed and piloted, consisting of questions on pain medication, symptoms and items 29 and 30 from the European Organisation for Research and Treatment of Cancer (EORTC) QLQ-C30 which measure global HRQoL [11]. The full interview schedule is available as an online supplement.

In the section on pain medication, patients were first asked open questions to be answered from memory (1) about the medications they used for pain control, both at the time of the interview as well as in the past; (2) for which pain these medications were used; (3) the side effects which patients experienced as being directly attributable to the identified analgesic medications; (4) any side effects whose attribution they were uncertain of; (5) whether they felt limited in daily activities because of these side effects; (6) whether they ever discontinued an analgesic because of its side effects and if so, which side effect caused them to discontinue the medicine. Only analgesics used for pain caused directly by myeloma or by disease-modifying therapy, for example chemotherapyinduced painful peripheral neuropathy, were considered relevant. For the patients' clarity, we included codeine and co-codamol separately in the medication lists but in 
the results, we combined the responses for these two medications under 'codeine'.

After this first section of the interview, patients were shown prompt lists containing the most common analgesics and their common side effects. They were asked again about their history of pain medication and experienced side effects. Using this procedure, we were able to compare the ability of patients to recall their analgesics and side effects accurately by comparing the answers given from memory with the items they had recognized after being prompted by the lists.

Patients were asked to give their overall opinion regarding the presence and influence of side effects of analgesics on their HRQoL ('Yes, in a positive way'; 'Yes, in a negative way'; 'No, not at all'.) Patients also answered questions 29 and 30 of the EORTC QLQ-C30 questionnaire, which have responses on a 7-point Likert scale ranging from 1 ('very poor overall health/QoL') to 7 ('excellent overall health/QoL'). The resulting score can range from 2 to 14 , which was transformed to a $0-100$ scale, according to EORTC recommendations. Together, these two questions capture a highly personal view of the patients own perception of global HRQoL using an instrument that has been translated and validated into 81 languages and has been used in more than 3,000 studies worldwide [11, 12]. Patients were asked these questions in two stages: first, for their present situation (which would have included the impact of any side effects) and next, for the hypothetical situation in which they were asked to estimate what their HRQoL would have been, if they did not experience any of their recognised analgesic side effects.

The draft interview was pilot-tested with five health care specialists and one patient representative to assess applicability and comprehensibility, and adapted accordingly. Subsequently, the adapted questionnaire was piloted on two members of the North Trent Consumer Research Panel and finalised after responding to their feedback.

In the main study, patients were identified from the day ward, in-patient wards and out-patient clinics. Consecutive patients were screened to reduce selection bias. Eligible patients received an information sheet about the procedure and had the opportunity to ask questions for clarification. They were recruited to the study after signing an informed consent form. Interviews were conducted by SS with help from JB.

Statistical analysis

Data were analysed with SPSS, version 15.0. As this was an observational cross-sectional study, most of the results are presented as descriptive. To calculate the difference between global HRQoL-values with and without side effects, Student's $t$ tests for paired samples were used.

\section{Results}

Sample

Between October and December 2008, 73 MM patients were approached. Forty-six patients were excluded from the interview because they did not use any other pain medication than paracetamol or were only on bisphosphonates or corticosteroids. Five other patients declined to take part, because they had already recently taken part in other studies $(n=2)$; did not feel well enough to take part $(n=2)$ or for no given reason $(n=1)$. See CONSORT diagram, Fig. 1.

Twenty-two patients with a mean(SD) time since diagnosis of 3.3(1.8)years agreed to take part in the interview. Other patient characteristics are given in Table 1. One patient withdrew after starting. The interview took a mean(SD) time of 25(7.28)min.

Pain medications

Analgesics were most often used for back or other bony pain caused by MM. Some patients additionally needed pain relief for treatment-related neuropathic pain in their lower extremities. The majority (17/21) were on step 3 of the WHO analgesic ladder, i.e., they were using a 'strong' opioid with or without other non-opioid analgesics. The most frequently used current pain medications were paracetamol $(n=16)$, followed by fentanyl patches $(n=10)$ and oxycodone $(n=7)$. The most frequently previously used drugs were morphine $(n=7)$, followed by gabapentin $(n=6)$ and codeine $(n=6)$. See Table 2.

When asked what analgesics they used now and had previously been using, patients were able to recall a median of the two (range 0-4) analgesics. When they were prompted

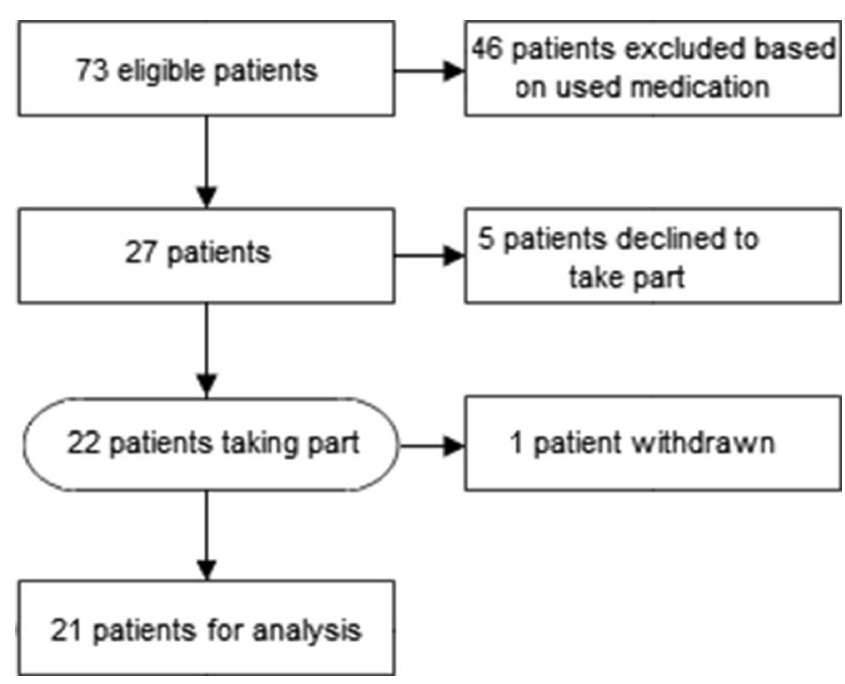

Fig. 1 Patient disposition (CONSORT diagram) 
by a list of common pain medications, they were now able to recognize a median of the three (range 1-7) analgesics. Only one patient reported, without prompting, taking a bisphosphonate for pain control and none associated the use of corticosteroids as an analgesic. With the help of the prompt sheet, seven patients recalled using or having used bisphosphonates, while four patients recalled using or having used corticosteroids.

\section{Side effects}

The median number of freely recalled current and past side effects, attributed to their pain medication, was two (range 1-5). Patients declared a median of zero (range 0-3) side effects of unknown origin, which might or might not have been caused by analgesics. When patients were asked to read through the prompt sheet with the most common side effects arising from pain medication, some were now able to identify more, giving a median of two (range 1-8) side effects as attributed to their analgesics. They also now identified a further median of one (range 0-7) side effects of unknown origin. Altogether, the 21 patients reported a range of 52 specific side effects from pain medication, which could be classified into 18 distinct groups. Additionally, they mentioned a total of 42 specific side effects of unknown origin.

Table 1 Patient characteristics

Patients $(n=21)$

\begin{tabular}{ll}
\hline Gender & \\
Male & 10 \\
Female & 11 \\
Mean age $( \pm$ SD) in years & $65(9)$ \\
Mean time since diagnosis \pm SD in years & $3.3(1.8)$ \\
WHO Performance status & \\
0 & 0 \\
1 & 1 \\
2 & 11 \\
3 & 7 \\
4 & 2 \\
Analgesia according to WHO Pain ladder & \\
1 & 0 \\
2 & 4 \\
3 & 17 \\
Number of analgesic drugs used per patient & \\
1 & 2 \\
2 & 5 \\
3 & 8 \\
4 & 2 \\
\hline
\end{tabular}

Table 2 Pain medications used in 21 patients with multiple myeloma: at present, in the past and cumulative usage

\begin{tabular}{|c|c|c|c|c|c|c|}
\hline \multirow[t]{2}{*}{ Pain medication } & \multicolumn{2}{|c|}{ At present } & \multicolumn{2}{|c|}{ In the past } & \multicolumn{2}{|c|}{ Cumulative } \\
\hline & $n$ & $\%$ & $n$ & $\%$ & $n$ & $\%$ \\
\hline Paracetamol & 15 & 71 & 4 & 19 & 19 & 90 \\
\hline Fentanyl (patch) & 10 & 48 & 1 & 5 & 11 & 52 \\
\hline Morphine & 3 & 14 & 7 & 33 & 10 & 48 \\
\hline Oxycodone & 7 & 33 & 2 & 10 & 9 & 43 \\
\hline Gabapentin & 3 & 14 & 6 & 29 & 9 & 43 \\
\hline Codeine & 3 & 14 & 6 & 29 & 9 & 43 \\
\hline Bisphosphonates & 7 & 33 & 1 & 5 & 8 & 38 \\
\hline Tramadol & 3 & 14 & 3 & 14 & 6 & 29 \\
\hline Pregabalin & 3 & 14 & 1 & 5 & 4 & 19 \\
\hline Corticosteroids & 2 & 10 & 2 & 10 & 4 & 19 \\
\hline Aspirin & 2 & 10 & 2 & 10 & 4 & 19 \\
\hline Fentanyl (lozenge) & 1 & 5 & 2 & 10 & 3 & 14 \\
\hline Methadone & 2 & 10 & 0 & 0 & 2 & 10 \\
\hline Buprenorphine (patch) & 2 & 10 & 0 & 0 & 2 & 10 \\
\hline Ketamine & 1 & 5 & 0 & 0 & 1 & 5 \\
\hline Diclofenac & 1 & 5 & 0 & 0 & 1 & 5 \\
\hline Diamorphine & 0 & 0 & 1 & 5 & 1 & 5 \\
\hline Dihydrocodeine & 0 & 0 & 1 & 5 & 1 & 5 \\
\hline
\end{tabular}

The most commonly reported side effects caused by pain medication were constipation $(n=10)$, dizziness $(n=8)$ and tiredness/fatigue $(n=8)$. The most common side effects of unknown origin were constipation $(n=8)$ and increased sweating $(n=5)$. See Table 3.

The majority of the 52 side effects attributable to pain medications were rated as severe $(n=25)$, followed by moderate $(n=18)$ and mild $(n=9)$. Out of the 42 side effects of unknown origin, the majority was rated as moderate $(n=16)$, followed by mild $(n=14)$ and severe $(n=12)$. See Table 4.

None of the patients mentioned a side effect that is not known to be potentially related to the prescribed pain medications [13].

In absolute numbers, fentanyl patches were responsible for the largest number of reported side effects $(n=12$ in 11 patients receiving this drug, currently and in the past), followed by codeine ( $n=9$ in nine patients), morphine ( $n=$ 8 in ten patients) and oxycodone ( $n=5$ in seven patients). In relative numbers, methadone was responsible for the highest frequency of side effects, with four side effect reports by the two patients, followed by fentanyl (12 side effects in 11 patients) and codeine (nine side effects in nine patients).

Ten patients (48\%) reported that, at some point during their illness, they stopped taking pain medication or had cut down on the dose of their medication, because of side effects, most often with codeine $(n=3)$. 
Table 3 Side effects of medication in multiple myeloma patients: Those due to analgesics, those due to unknown medication and cumulative side effects

\begin{tabular}{|c|c|c|c|}
\hline Side effects & $\begin{array}{l}\text { Pain medication } \\
n\end{array}$ & $\begin{array}{l}\text { Unknown cause } \\
n\end{array}$ & $\begin{array}{l}\text { Cumulative } \\
n\end{array}$ \\
\hline Constipation & 10 & 7 & 17 \\
\hline Tiredness/fatigue & 8 & 4 & 12 \\
\hline Dizziness & 8 & 1 & 9 \\
\hline Drowsiness & 5 & 4 & 9 \\
\hline Hallucinations & 4 & 1 & 5 \\
\hline Nausea & 1 & 4 & 5 \\
\hline Increased sweating & 0 & 5 & 5 \\
\hline Feeling sad, depressed & 3 & 1 & 4 \\
\hline Jerky movements & 2 & 2 & 4 \\
\hline Dry mouth & 1 & 3 & 4 \\
\hline Vomiting & 2 & 1 & 3 \\
\hline Loss of interest in sex & 1 & 2 & 3 \\
\hline Problems passing urine & 0 & 3 & 3 \\
\hline Itching & 1 & 1 & 2 \\
\hline ONJ & 1 & 0 & 1 \\
\hline Flatulence & 1 & 0 & 1 \\
\hline Withdrawal & 1 & 0 & 1 \\
\hline Hot flushes & 1 & 0 & 1 \\
\hline Flu symptoms & 1 & 0 & 1 \\
\hline Swelling & 1 & 0 & 1 \\
\hline Indigestion/heartburn & 0 & 1 & 1 \\
\hline Itching & 0 & 1 & 1 \\
\hline Skin rash & 0 & 1 & 1 \\
\hline Total & 52 & 42 & 94 \\
\hline
\end{tabular}

ONJ Osteonecrosis of the jaw

The majority of patients $(n=14 ; 67 \%)$ reported that the side effects did not prevent them from performing daily activities, although they often reported a reduced level as compared to their activities before their illness.

Global health-related quality of life

Eight patients ( $38 \%)$ thought that the side effects from their pain medication had a negative impact on their global

Table 4 Severity of medication side effects experienced by patients with multiple myeloma: Side effects from analgesics, from unknown medication and cumulative side effects

\begin{tabular}{llll}
\hline Severity of side effects & $\begin{array}{l}\text { Pain medication } \\
n\end{array}$ & $\begin{array}{l}\text { Unknown cause } \\
n\end{array}$ & $\begin{array}{l}\text { Cumulative } \\
n\end{array}$ \\
\hline Mild & 9 & 14 & 23 \\
Moderate & 18 & 16 & 34 \\
Severe & 25 & 12 & 37 \\
Total & 52 & 42 & 94 \\
\hline
\end{tabular}

HRQoL, whereas 13 patients (62\%) did not think they had an impact.

The actual current global HRQoL was mean (range) 48.3 (38.7-57.9) out of a 100. The estimated mean global HRQoL assuming the absence of side effects from the analgesics was $62.6(53.5-71.7)$, the difference being $14.3(p=0.001)$ (see Table 5). A difference of $>10$ in the EORTC QLQ-C30 scales is regarded as being clinically significant [14].

\section{Discussion}

MM is a chronic malignant disease which is ultimately fatal, but with recent improvements in disease management, including biological therapies and haematopoeitic stem cell transplantation (HSCT), patients can now expect to live for many years [1]. However, the pain of bone lesions and additional treatment-emergent complications such as peripheral neuropathy can impose a significant burden on survivors [2]. We have recently shown that even 5 years after diagnosis and after three lines of treatments, patients reported pain and fatigue as their most pressing symptoms; pain was neuropathic in half the subjects [15]. It is now acknowledged in evidence-based guidelines of MM that supportive care has a significant role in its management [3].

One of the most important aspects of supportive care in $\mathrm{MM}$ is to provide effective and safe pain relief. The results of this study suggest that the side effects of analgesics may significantly reduce HRQoL of patients with symptomatic MM. Thus, despite the intention of supportive care to optimise disease management and minimise side effects, HRQoL may be inadvertently compromised of the prescription of analgesic medications. These findings concur with the previous cross-sectional studies in patients which estimated side effects of opioids in mixed groups of cancer patients. Villars et al. (2007) studied oncology outpatients with bone metastases and found a correlation between total dose of opioid and several reported side effects [10]. The majority of their subjects had solid tumours were having forms of anti-cancer treatment and less than $60 \%$ had cancer for longer than 1 year. Subjects studied by Glare et al. (2006) were in a palliative care programme, had predominantly solid tumours and the majority $(74 \%)$ were of poor performance status (Eastern Cooperative Oncology Group [ECOG] 3-4) [16]. They found the side effects rated as 'moderate to severe' in this population to be dry mouth, sedation, anorexia, seating, constipation, urinary hesitancy and nightmares. We believe ours is the only study to describe analgesic side effects in a homogeneous diagnostic group of patients who were long-term ambulatory survivors of cancer. 
Table 5 Self-rated overall quality of life in multiple myeloma patients ${ }^{1}$

HRQoL scores $(95 \% \mathrm{CI})^{2}$

Current-including current medication side-effects

$48.3(38.7-57.9)$

Estimated-excluding current medication side-effects

$62.6(53.5-71.7)$

${ }^{1}$ The difference in means between 'Including side-effects' and 'Excluding side-effects' was highly significant, $p=0.001$ (Student's $t$ test)

${ }^{2}$ Overall health-related quality of life taken from Items 29 ('Overall health') and 30 ('Overall quality of life') from European Organisation for Research and Treatment of Cancer (EORTC) QLQ-C30 on a scale from $0-100$

Earlier studies of HRQoL in MM have focused on the impact of increasingly more intensive disease-modifying treatments in $\mathrm{MM}[17,18]$. We found no previous quantitative studies on the prevalence and impact of adverse effects of analgesics on HRQoL in this disease. This study has therefore provided a first insight into the evaluation of HRQoL in relation to the side effects from analgesics experienced by patients with symptomatic MM. We found that analgesic side effects were rated by patients as mostly 'severe'. It is noteworthy that although the perceived difference in HRQoL with and without analgesic side effects was 14.3, which would be regarded as clinically significant in the literature, this was not the initial impression from the question "Do the side effects from your pain medication have an effect on your quality of life?'. The majority (62\%) replied 'No' and only $38 \%$ stated it had a negative influence. This shows that asking a single global question with binary responses could give a superficially reassuring answer, which is belied by more detailed structured questioning as the use of the EORTC QLQ-C30 items 29+30 showed. Further evidence that HRQoL had indeed been affected by analgesic side effects came from the observation that nearly half the sample had at some time stopped or reduced analgesic drugs because of them. Modern medicine offers many solutions and options to bring this number down, education being the first and foremost of them [8].

Methadone was the opioid associated with the highest absolute number of side effects, although this may be skewed because of the small sample size. Side effects were also common with fentanyl patches and rather more frequently with fentanyl than with morphine. This is surprising in view of systematic reviews and meta-analyses demonstrating that fentanyl causes significantly less side effects compared with morphine [19, 20]. Prior to being on fentanyl, patients most commonly had used morphine and oxycodone. It is possible that the first exposure to these prior opioids had already established a memory of side effects in these patients; second, the commonest reason for switching from morphine or oxycodone to fentanyl would have been intolerance to their side effects; and third, long-term opioid use led to fentanyl doses being relatively higher. Unfortunately, we did not record doses of drugs taken currently or in the past.

Consistent with the literature on chronic opioid use, we found constipation to be the most prevalent side effect. Apart from its detrimental effect on wellbeing, opioid-induced constipation (OIC) has a significant health economic cost to society [21]. Unfortunately, the evidence base for current management of OIC using conventional laxatives is poor [22]. Only the newer peripherally acting opioid antagonist drugs have been shown in large double-blind RCTs to be efficacious without compromising pain control [23].

Other studies have confirmed that analgesics and especially opioids are associated with significant adverse effects, not only for cancer and palliative care patients, but also in the general population and especially in older people [10]. Zhang et al. (2009) reported from an Australian adverse reaction (ADR) database that 'analgesics/antipyretics/anti-inflammatory drugs' as a class were associated with the second highest proportion (after cardiovascular agents) responsible for first time ADR hospital admission, causing $16.4 \%$ of admissions in people over 60 years [24]. Opioids were responsible for $4.9 \%$ of these admissions in older patients.

Side effects may reduce compliance and therefore effectiveness of analgesic therapy. Gregorian et al. (2010) demonstrated using adaptive conjoint analysis that patients with both cancer and non-cancer chronic pain were able to trade off a degree of pain control against the relative bothersomeness of opioid-induced side effects [25]. The highest amount of pain relief that patients were willing to trade off was in the order of 2 points on an 11-point scale with respect to nausea and vomiting.

We did not find any previous studies that tried to assess the impact of analgesic side effects on self-reported HRQoL. Schmier et al. (2002) used a conjoint analysis methodology to assess the relative utility states of degrees of pain control balanced by levels of side effects [26]. Interestingly, their cohort of cancer patients $(n=25)$ reported a mean current global QoL rating on the EORTC QLQ-C30 of 48.3 (SD; 21), which was exactly the same as the patients reported in our study, namely mean 48.3 (95\% CI; 38.7-57.9). In their study, patients were able to declare a reduction in utility of 5 points for a change in side effect severity from mild to severe, with pain control held constant.

Glintborg et al. (2007) have shown that Danish patients' reports of their drug history corresponded poorly with hospital pharmacy records for those patients [27]. In that study, patients reported a median (range) of four (0-14) prescription drugs whilst pharmacy records gave one $(0-11)$. These patients failed to report $27 \%$ of drugs they were taking on admission. Thus, patients' own recall of drug histories is unreliable and needs to be corroborated with external records. We confirm this with our patients under-reporting the number 
of analgesic drugs from memory, compared to checking them on a prompt list, although we did not match them to pharmacy records.

\section{Study limitations}

This study has a number of limitations, which means the results should be interpreted cautiously. The first is the small sample size - further studies with a larger number of patients will be needed to confirm or refute these findings. Although we believe the consecutive sampling from haematology clinic, day ward and in-patients helped to reduce selection bias, we cannot be sure that the final sample is representative of other haematology services. Further multi-centre studies would be needed to ensure generalizability of the findings.

Another limitation is the possibility of interviewer and interviewee bias. To avoid interviewer bias, the interviews were all conducted by the same two individuals. Interviewee bias was minimised by selecting consecutive MM patients attending day ward, out-patients or admitted to the hospital with effectively only lack of fluency in English being an exclusion factor.

Some patients expressed difficulties in identifying and distinguishing between side effects caused by pain medication, disease-directed therapy or symptoms of disease itself. We used a standardised prompt sheet to aid patients' recall of side effects. However, when being faced by the prompt sheet, some patients may have overestimated them. Similarly, the use of medication prompt sheets could have led to overestimation of recall of previous analgesic drugs. We did not investigate whether each mentioned side effect matched the exact drug to which this side effect was attributed, as this would have been too extensive for the resources of this service review. Overall, however, because patients seem to be able to recognize more side effects from a prompt list than they could freely recall, providing them with a standard screening symptom list when monitoring and assessing their side effects in clinical follow-up may be useful in providing better routine supportive care. In future research studies, these problems could be solved by prospective recording of drugs and side effects as they emerge. Patients could be asked to keep diaries to record side effects and their daily impact.

Another possible weakness is that our findings could have been influenced by other factors such as symptoms of MM disease progression and co-morbidity that may mimic side effects of painkillers and thus impact on HRQoL. The number of patients included in this study was too small to quantify this problem.

Because we were obtaining drug histories from patients directly and did not check these against their case-notes and drug charts, we were unable to collect data on drug doses. In future studies of analgesic side effects, current drug doses should be collected as well as information on other nonanalgesic drugs which could be causing side effects that overlap with analgesic side effects. Furthermore, the study could have benefited from a control group, e.g., the MM patients who were excluded because they only used bisphosphonates, corticosteroids and paracetamol.

We asked patients to compare their current quality of life with an estimated quality of life if they did not have analgesic side effects. Only a few patients expressed difficulties in imagining the scenario in which they had MM, were using pain medication, but did not experience any side effects. Ideally, we should have measured self-rated HRQoL prospectively before and after patients started specific analgesic drugs. However, we believe that for the majority of patients this approach was able to quantify a realistic estimate-from the patients' own perspective — of impact of analgesics on quality of life.

\section{Conclusion and recommendations}

Even when patients' self-assessment may be clouded by the effects of MM disease and its treatment and given the difficulty of answering specific questions for some, we can state with some confidence that analgesic side effects, in spite of the measures used in supportive care to minimise them, constitute a major problem in MM management [2, 3]. This service review indicates that further research using prospective studies on pain management and side effects of analgesics should be conducted in patients with MM and also in other diseases where long-term, and especially opioid, analgesics are used.

This service evaluation can be seen as a semi-quantitative pilot project which has given sufficient preliminary information to justify a larger research study. In order to increase sample size and therefore generalizability and to tackle many of the acknowledged limitations, our intensive cross-sectional interview-based design should be converted to a self-rated prospective questionnaire-based survey with parallel recording of drugs and doses from pharmacy records.

Funding source SS was funded by an Erasmus scholarship.

Contributions SS, SHA, JB, JAS, YEn designed the study. SS and JB collected data. YEz and JAS identified patients. AG and TG were members of North Trent Consumer Research Panel. LC was member of the institutional Clinical Effectiveness Unit. SS, SHA, JAS, JB, SV, KV wrote the manuscript. SS performed this study as a medical student of Nijmegen University Medical School, Netherlands. SHA was her research supervisor and YE was her academic supervisor.

We would like to acknowledge statistical advice from Dr Stephen Walters, ScHARR, The University of Sheffield.

Copyright The Corresponding Author has the right to grant on behalf of all authors and does grant on behalf of all authors, a worldwide licence to the Publishers and its licensees in perpetuity, in all forms, formats and media (whether known now or created in the future), to (1) publish, reproduce, distribute, display and store the Contribution, (2) translate 
the Contribution into other languages, create adaptations, reprints, include within collections and create summaries, extracts and/or, abstracts of the Contribution, (3) create any other derivative work(s) based on the Contribution, (4) to exploit all subsidiary rights in the Contribution, (5) the inclusion of electronic links from the Contribution to third party material where-ever it may be located; and (6) licence any third party to do any or all of the above.

Transparency declaration The lead author (the manuscript's guarantor) affirms that this manuscript is an honest, accurate, and transparent account of the study being reported; that no important aspects of the study have been omitted; and that any discrepancies from the study as planned (and, if relevant, registered) have been explained.

Conflict of interests All authors have completed the ICMJE uniform disclosure form at www.icmje.org/coi_disclosure.pdf and declare: No support from any organisation for the submitted work; no financial relationships with any organisations that might have an interest in the submitted work in the previous three years; no other relationships or activities that could appear to have influenced the submitted work.

Open Access This article is distributed under the terms of the Creative Commons Attribution Noncommercial License which permits any noncommercial use, distribution, and reproduction in any medium, provided the original author(s) and the source are credited.

\section{References}

1. Bird JM, Owen RG, D'Sa S et al (2011) Guidelines for the diagnosis and management of multiple myeloma 2011. Br J Haematol 154:32-75

2. Niscola P, Scaramucci L, Romani C et al (2010) Pain management in multiple myeloma. Expert Rev Anticancer Ther 10:415-425

3. Snowden JA, Ahmedzai SH, Ashcroft J et al (2011) Guidelines for supportive care in multiple myeloma 2011. Br J Haematol 154:76103

4. Mhaskar R, Redzepovic J, Wheatley K, Clark OA, Miladinovic B, Glasmacher A, Kumar A, Djulbegovic B (2010) Bisphosphonates in multiple myeloma. Cochrane Database Syst Rev 5:CD003188. doi: 10.1002/14651858.CD003188

5. Mendoza TR, Koyyalagunta D, Burton AW et al (2012) Changes in pain and other symptoms in patients with painful multiple myelomarelated vertebral fracture treated with kyphoplasty or vertebroplasty. J Pain : Off J Am Pain Soc 13:564-570

6. Wedin R (2001) Surgical treatment for pathologic fracture. Acta Orthop Scand Suppl 72(2p):1-29

7. Cartoni C, Brunetti GA, Federico V et al (2012) Controlled-release oxycodone for the treatment of bortezomib-induced neuropathic pain in patients with multiple myeloma. Support Care Cancer : Off J Multinatl Assoc Support Care Cancer 20:2621-2626

8. Ahmedzai SH (2001) Window of opportunity for pain control in the terminally ill. Lancet 357:1304-1305

9. Weiss SC, Emanuel LL, Fairclough DL, Emanuel EJ (2001) Understanding the experience of pain in terminally ill patients. Lancet 357:1311-1315

10. Villars P, Dodd M, West C et al (2007) Differences in the prevalence and severity of side effects based on type of analgesic prescription in patients with chronic cancer pain. J Pain Symptom Manag 33:67-77

11. Aaronson NK, Ahmedzai S, Bergman B et al (1993) The European Organization for Research and Treatment of Cancer QLQ-C30: a quality-of-life instrument for use in international clinical trials in oncology. J Natl Cancer Inst 85:365-376

12. http://groups.eortc.be/qol/eortc-qlq-c30 European Organsiation for Research and Treatment of Cancer - Quality of Life Department. (Accessed March 29th, 2014)

13. Dickman A (2012) Drugs in palliative care, 2nd edn. Oxford University Press. http://ukcatalogue.oup.com/product/ 9780199660391.do

14. Osoba D (2002) A taxonomy of the uses of health-related quality-oflife instruments in cancer care and the clinical meaningfulness of the results. Med Care 40:54-55

15. Boland E, Eiser C, Ezaydi Y, Greenfield DM, Ahmedzai SH, Snowden JA (2013) Living with advanced but stable multiple myeloma: a study of the symptom burden and cumulative effects of disease and intensive (hematopoietic stem cell transplant-based) treatment on health-related quality of life. J Pain Symptom Manag 46:671-680

16. Glare P, Walsh D, Sheehan D (2006) The adverse effects of morphine: a prospective survey of common symptoms during repeated dosing for chronic cancer pain. Am J Hosp Palliat Care 23:229-235

17. Gulbrandsen N, Wisloff F, Brinch L et al (2001) Health-related quality of life in multiple myeloma patients receiving high-dose chemotherapy with autologous blood stem-cell support. Med Oncol 18:65-77

18. Uyl-de Groot CA, Buijt I, Gloudemans IJ, Ossenkoppele GJ, Berg HP, Huijgens PC (2005) Health related quality of life in patients with multiple myeloma undergoing a double transplantation. Eur J Haematol 74:136-143

19. Tassinari D, Sartori S, Tamburini E et al (2008) Adverse effects of transdermal opiates treating moderate-severe cancer pain in comparison to long-acting morphine: a meta-analysis and systematic review of the literature. J Palliat Med 11:492-501

20. Clark AJ, Ahmedzai SH, Allan LG et al (2004) Efficacy and safety of transdermal fentanyl and sustained-release oral morphine in patients with cancer and chronic non-cancer pain. Curr Med Res Opin 20: $1419-1428$

21. Hjalte F, Berggren AC, Bergendahl H, Hjortsberg C (2010) The direct and indirect costs of opioid-induced constipation. J Pain Symptom Manag 40:696-703

22. Ahmedzai SH, Boland J. (2010) Constipation in people prescribed opioids. Clin Evid. PMC2907601

23. Ahmedzai SH, Nauck F, Bar-Sela G, Bosse B, Leyendecker P, Hopp M (2012) A randomized, double-blind, active-controlled, double-dummy, parallel-group study to determine the safety and efficacy of oxycodone/naloxone prolonged-release tablets in patients with moderate/severe, chronic cancer pain. Palliat Med 26:50-60

24. Zhang M, Holman CD, Price SD, Sanfilippo FM, Preen DB, Bulsara MK (2009) Comorbidity and repeat admission to hospital for adverse drug reactions in older adults: Retrospective cohort study. BMJ 338: a2752

25. Gregorian RS Jr, Gasik A, Kwong WJ, Voeller S, Kavanagh S (2010) Importance of side effects in opioid treatment: a trade-off analysis with patients and physicians. J Pain : Off J Am Pain Soc 11:10951108

26. Schmier JK, Palmer CS, Flood EM, Gourlay G (2002) Utility assessments of opioid treatment for chronic pain. Pain Med 3: 218-230

27. Glintborg B, Poulsen HE, Dalhoff KP (2008) The use of nationwide on-line prescription records improves the drug history in hospitalized patients. Br J Clin Pharmacol 65:265-269 